\title{
2020 Reviewer Acknowledgement
}

\section{Editorial Office of Journal of Exploratory Research in Pharmacology}

We thank the following reviewers for their contribution and support in 2020 .

John J. Araujo

Columbia

Anthony Au

Malaysia

Priyamadhaba Behera

India

Giancarlo Ceccarelli Italy

Kishore B. Challagundla USA

Sy Giin Chong Ireland

JK Dawson UK

Giovanni Battista Dell'Isola Italy

Lucas D Dias Brazil

Talha Bin Emran Pakistan

Junjiang Fu China

Owen Rees Griffiths UK

Xinsheng Gu China

Surampalli Gurunath India

Darlan Gusso
Brazil
Kadda Hachem
Algeria
Idress Hamad Attitalla
Libya
Nafissa Ismail
Canada
Saurav Kar
India
Tom C Karagiannis
USA
Nicholas Kipshidze
USA
Xin Li
China
Jingting Li
USA
Xiao-Hong Li
USA
Yang Liu
China
Cyprian Ogbonna Onyeji
Nigeria
Minhua Peng
China
Xingshun Qi
China

Hakim Rahmoune Algeria

Luis J Ramirez-Jirano Mexico

Muhammad Shahid Pakistan

Gurpreet Singh India

Luca Steardo Italy

Bing Sun USA

Abilo Tadesse Ethiopia

Hemant D. Une India

Srijayaprakash Babu Uppada USA

Lili Wang USA

Karol Wróblewski Poland

Jie Wu USA

Jianshe Yang China

Lili Zhou China

DOI: 10.14218/JERP.2020.000RA 